\title{
Plasma Fatty Acid Composition and Incident Ischemic Stroke in Middle-Aged Adults: The Atherosclerosis Risk in Communities (ARIC) Study
}

\author{
Kazumasa Yamagishi ${ }^{a}$ b $\quad$ Aaron R. Folsom ${ }^{a}$ Lyn M. Steffen ${ }^{a}$ \\ for the ARIC Study Investigators \\ a Division of Epidemiology and Community Health, School of Public Health, University of Minnesota, \\ Minneapolis, Minn., USA; ${ }^{b}$ Department of Public Health Medicine, Faculty of Medicine, University of Tsukuba, \\ Tsukuba, Japan
}

\author{
Key Words \\ Longitudinal study · Epidemiology · Fat · Biomarkers . \\ Risk factors
}

\begin{abstract}
Background: The association of individual fatty acids with ischemic stroke has not been thoroughly studied, and results have been inconsistent. Few prospective studies have systematically explored the association of biomarkers of fatty acid intake with stroke. The aim of this study was to explore which individual plasma fatty acids would be associated with higher risk of ischemic stroke among whites. Methods: We studied 3,870 white men and women from the Minneapolis field center of the Atherosclerosis Risk in Communities (ARIC) Study, aged 45-64 years at baseline (1987-1989), who had plasma cholesterol ester (CE) and phospholipid (PL) fatty acids measured. Participants were followed through 2008 for incident ischemic stroke. Hazard ratios (HRs) with $95 \%$ confidence intervals (Cls) across quartiles of each fatty acid, measured as the percentage of total fatty acids, were calculated using the Cox proportional hazards model. Results: During a maximum of 22 years of follow-up, we identified
\end{abstract}

168 cases of ischemic stroke. After adjustment for age and sex, plasma levels of saturated fatty acids were associated positively: $\mathrm{HR}(95 \% \mathrm{Cl})$ of the highest versus the lowest quartile for CE fraction was $1.93(1.23-3.04$, p for trend $=0.01)$ and that for PL fraction was $1.64(1.05-2.57, \mathrm{p}$ for trend $=0.03)$. There was also a positive linear association with monounsaturated fatty acids, especially with palmitoleic acid: HR $(95 \% \mathrm{Cl})$ of the highest versus the lowest quartile for CE fraction was $1.86(1.20-2.87, \mathrm{p}$ for trend $=0.003)$ and that for $\mathrm{PL}$ fraction was $1.52(0.99-2.34, p$ for trend $=0.005)$. No associations of $\omega-3$ and $\omega-6$ polyunsaturated fatty acids with ischemic stroke were observed, but linoleic acid was inversely and nonlinearly associated with ischemic stroke: HR $(95 \% \mathrm{Cl})$ of the highest versus the lowest quartile for $C E$ fraction was $0.64(0.43-0.97, \mathrm{p}$ for trend $=0.13)$ and that for PL fraction was $0.69(0.45-1.05, p$ for trend $=0.24)$. These associations were generally unchanged after adjustment for cardiovascular risk factors. Conclusions: In this US cohort of whites, we found significant positive associations of plasma saturated and monounsaturated fatty acids, especially of palmitoleic acid, with ischemic stroke. We also found an inverse nonlinear association between linoleic acid and ischemic stroke.

Copyright $\odot 2013$ S. Karger AG, Basel

\section{KARGER}

E-Mail karger@karger.com

www.karger.com/ced (c) 2013 S. Karger AG, Basel

$1015-9770 / 13 / 0361-0038 \$ 38.00 / 0$
Aaron R. Folsom, MD, MPH

Division of Epidemiology and Community Health, School of Public Health

University of Minnesota, 1300 S. 2nd Street, Suite 300

Minneapolis, MN 55454-1015 (USA)

E-Mail folso001@umn.edu 


\section{Introduction}

It is well established that dietary intakes of fatty acids have a substantial impact on coronary disease; i.e. saturated fatty acids (SFA) increase and polyunsaturated fatty acids (PUFA) decrease serum cholesterol levels and risk of coronary disease [1]. However, the association of fatty acid intake with ischemic stroke has been less studied and results have been inconsistent [2-4]. Furthermore, few prospective studies have systematically explored the association of biomarkers of fatty acid intake with stroke [5-8].

In this study, we examined the association between plasma fatty acid composition and incident ischemic stroke in participants recruited from the Minneapolis field center of the Atherosclerosis Risk in Communities (ARIC) Study, where plasma cholesterol ester (CE) and phospholipid (PL) fatty acids were measured. Generally, plasma fatty acids are considered good biomarkers for estimating recent dietary fatty acid intake [9], but are also affected by endogenous synthesis influenced by genetic variation, intrauterine programming and other lifestyle factors $[10,11]$. Several desaturases, $\Delta-9-, \Delta-6$ and $\Delta$-5-desaturases, are known to be involved in endogenous fatty acid synthesis and affected by both diet and genetics [10]. They have been reported to be associated with several cardiovascular risk factors such as insulin resistance and the metabolic syndrome [12]. Therefore, it would also be of interest to know whether these enzyme activities are associated with risk of stroke. In the present study, we sought to explore which individual fatty acids in plasma, and which desaturase indices, are associated with risk of ischemic stroke in the US white population.

\section{Methods}

\section{Study Cohort}

The ARIC Study selected a population-based sample of 15,792 persons from 4 communities: Forsyth County, N.C., the city of Jackson, Miss., north-western suburbs of Minneapolis, Minn. and Washington County, Md., USA. Participants were 45-64 years of age during the baseline period (1987-1989). The ARIC study protocol was approved by each field center's institutional review board. After written informed consent was obtained, participants underwent a baseline clinical examination, as detailed elsewhere [13]. In the Minneapolis field center only $(n=4,009)$, baseline plasma was analyzed for fatty acids. We excluded persons who reported a self-reported history of stroke and/or transient ischemic attack at the baseline survey or those without plasma fatty acid data. We also excluded nonwhite subjects due to their small number. Our final sample included 1,859 men and 2,011 women.
Plasma Fatty Acid Measurement

Twelve-hour fasting blood was collected into $10-\mathrm{ml}$ vacuum tubes containing EDTA. Plasma was separated and dispensed into two $1.5-\mathrm{ml}$ aliquots and frozen at $-70^{\circ} \mathrm{C}$ for approximately 2 years before analysis for individual fatty acid composition by a single technician, who was blinded to stroke outcome status. The samples were only thawed once for the fatty acid measurements themselves. A previous study has shown that there were no significant changes in most fatty acids, regardless of lipid fraction, after $8-10$ years of $-80^{\circ} \mathrm{C}$ storage [14].

Details of plasma fatty acid measurement have been described elsewhere [15]. After separation of PL and CE fractions, the identity of 28 fatty acid peaks was ascertained by comparison of each peak's retention time shown by the gas-liquid chromatogram relative to the retention times of fatty acids in synthetic standards of known fatty acid composition. The percentage of each fatty acid among total fatty acids (\% total fatty acids) was quantified by the total area for all fatty acids. Absolute values of each fatty acid were not estimated. The data were electronically transferred from the gas chromatogram to a computer. Short-term test-retest reliability coefficients have been reported previously [16]; the values were as follows: SFA: $\mathrm{r}=0.70$ for $\mathrm{CE}$ and 0.57 for PL; monounsaturated fatty acids (MUFA): $r=0.71$ for CE and 0.46 for PL, and PUFA: $r=0.71$ for $\mathrm{CE}$ and 0.32 for PL. That report also provided long-term reliabilities [16], which were somewhat lower than short-term reliabilities. The correlations between plasma and dietary fatty acids measured by food frequency questionnaire have also been reported [15]: SFA: $r=$ 0.23 for CE and 0.15 for PL; PUFA: $r=0.31$ for CE and 0.25 for PL, and MUFA: $r=0.01$ for CE and 0.05 for PL, $p<0.01$ for $r>0.05$.

We examined several individual fatty acids in both CE and PL: myristic (14:0), pentadecanoic (15:0), palmitic (16:0), stearic (18: $0)$, palmitoleic $(16: 1, \omega 7)$, oleic $(18: 1, \omega 9)$, linoleic $(18: 2, \omega 6)$, $\gamma$-linoleic $(18: 3, \omega 6)$, dihomo- $\gamma$-linoleic $(20: 3, \omega 6)$, arachidonic $(20$ : $4, \omega 6)$, a-linoleic $(18: 3, \omega 3)$, eicosapentaenoic $(20: 5, \omega 3)$ and docosahexaenoic $(22: 6, \omega 3)$ acids. For the major fatty acid groups, we defined SFA as the sum of (12:0), (14:0), (15:0), (16:0), (18:0), (20: $0),(22: 0),(23: 0)$ and (24:0); MUFA as the sum of $(14: 1, \omega 5),(16:$ $1, \omega 7),(18: 1, \omega 9),(20: 1, \omega 9),(22: 1, \omega 9)$ and $(24: 1, \omega 9) ; \omega-6(n-6)$ PUFA as the sum of $(18: 2, \omega 6),(18: 3, \omega 6),(20: 2, \omega 6),(20: 3, \omega 6),(20$ : $4, \omega 6),(22: 4, \omega 6)$ and $(22: 5, \omega 6) ; \omega-3$ PUFA as the sum of $(18: 3, \omega 3)$, $(20: 3, \omega 3),(20: 5, \omega 3),(22: 5, \omega 3)$ and $(22: 6, \omega 3)$, and long-chain $\omega-3$ PUFA as the sum of $(20: 5, \omega 3),(22: 5, \omega 3)$ and $(22: 6, \omega 3)$.

\section{Ascertainment of Incident Ischemic Stroke Events}

Ischemic strokes were identified through annual telephone calls to participants to ascertain all hospitalizations in the past year, through review of local hospital discharge lists and via death certificates. Incident stroke was classified by a computer algorithm and physician review based on signs, symptoms, neuroimaging (CT/MRI) and other diagnostic reports, according to criteria adapted from the National Survey of Stroke [17]. A stroke was classified into definite or probable hospitalized ischemic (cardioembolic or thrombotic) or hemorrhagic stroke on the basis of neuroimaging studies and autopsy, when available. Definite and probable ischemic stroke, where a brain neuroimaging revealed acute infarction or showed no evidence of hemorrhage, was considered ischemic stroke. A definite thrombotic infarction was further classified into large-artery occlusive or lacunar, based on neuroimaging results. More details on the methods for ascertainment of incident stroke in ARIC have been given elsewhere [18]. 
Table 1. Sex-specific unadjusted baseline characteristics for those who did or did not develop ischemic stroke; ARIC Study, Minneapolis field center, 1987-1989

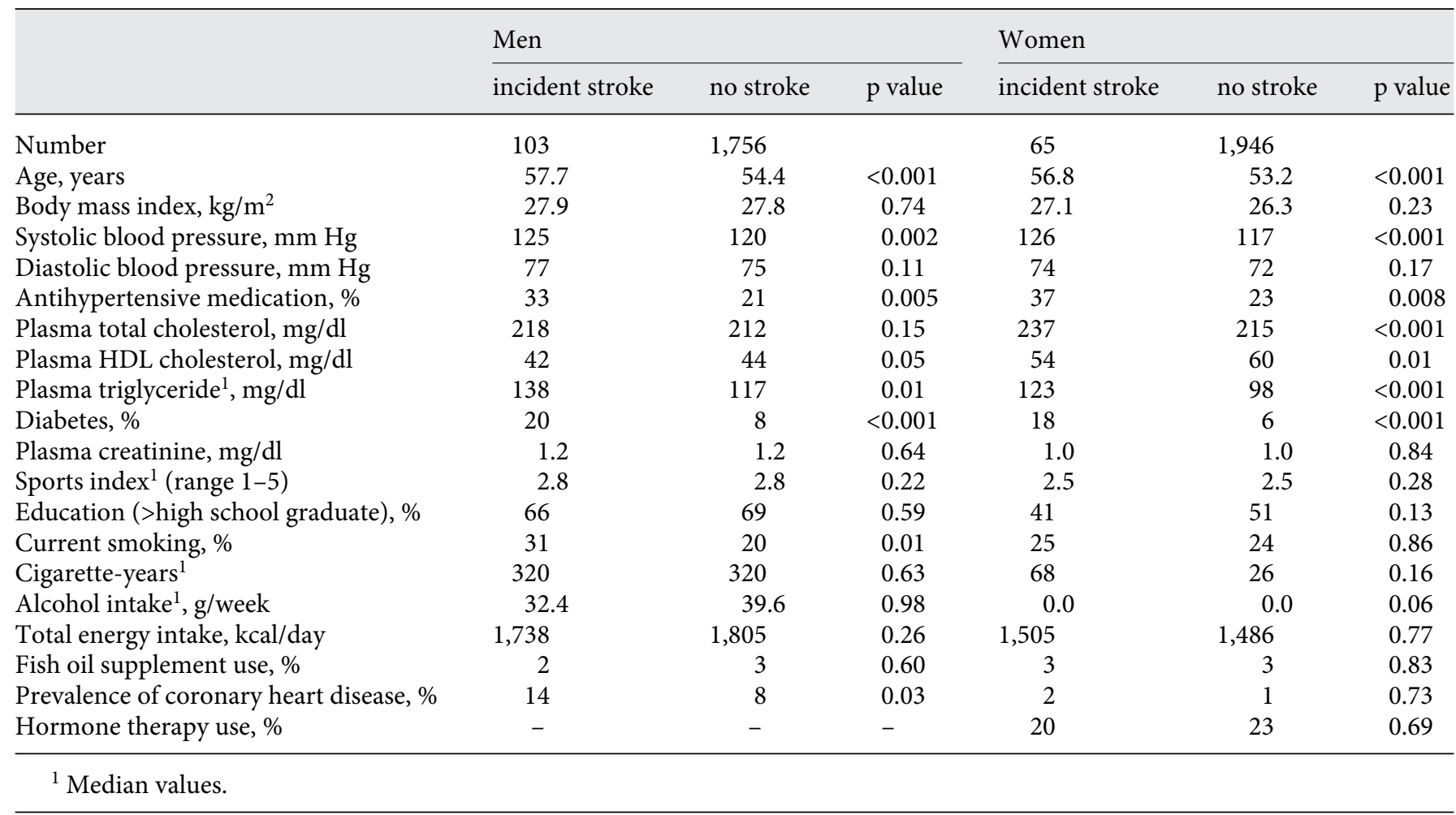

\section{Statistical Analysis}

For each participant, we calculated the person-years of followup from baseline in 1987-1989 to the first stroke endpoint, death, loss to follow-up, or the end of 2008. The median and maximum years of follow-up were 19.9 and 22.1, respectively. Hazard ratios (HRs) with 95\% confidence intervals (CIs) across quartiles of each fatty acid, measured as the percentage of total fatty acids, were calculated after adjustment in the Cox proportional hazards models for age and sex, and for multivariable adjustment models, smoking status (never, ex- or current smoker), cigarette-years and alcohol intake. We did not adjust for blood pressure, diabetes, plasma triglycerides or total or HDL cholesterol levels because we considered them possible mediators of any association between fatty acids and ischemic stroke. Factors that were tested but did not confound the associations included body mass index, education levels, sports index, hormone replacement therapy, energy intake and fish oil supplement use. The linear trend of HRs across the quartiles was tested using a variable with $-3,-1,1$ and 3 assigned to successive quartiles. Since there was no significant multiplicative interaction between sex and plasma fatty acids in relation to ischemic stroke, men and women were pooled. The proportional hazards assumption was tested using time by fatty acid interaction terms and was not violated except for myristic acid in the PL fraction and pentadecanoic acid in both fractions, for which the Cox analyses were further stratified by follow-up time ( $\leq 10$ and $>10$ years). Since no significant associations with ischemic stroke were found for either stratum, we only present pooled results.
In secondary analyses, we defined desaturase and elongase activity indices $[12,19]$ to examine the activity of each enzyme with incident ischemic stroke: the ratios of $(16: 1, \omega 7) /(16: 0)$ as the $\Delta-9$ desaturase index; $(18: 3, \omega 6) /(18: 2, \omega 6)$ as the $\Delta$-6-desaturase index for the CE fraction; $(20: 3, \omega 6) /(18: 2, \omega 6)$ as the $\Delta$-6-desaturase index for the PL fraction; $(20: 4, \omega 6) /(20: 3, \omega 6)$ as the $\Delta$-5-desaturase index, and (18:0)/(16:0) as the elongase index. Quartiles of each index were used in the analyses.

We used SAS version 9.1.3 Service Pack 4 (SAS Institute Inc., Cary, N.C., USA) for the analyses. All $\mathrm{p}$ values for statistical tests were two-tailed and values of $\mathrm{p}<0.05$ were regarded as statistically significant.

\section{Results}

During 70,735 person-years of follow-up of 3,870 persons (1,859 men and 2,011 women), we documented 168 incident ischemic stroke events (103 in men and 65 in women). Of these, there were 35 cardioembolic, 60 definite large-artery occlusive, 34 definite lacunar, and 39 unclassified/possible thrombotic infarctions.

Compared with those who had no ischemic stroke (table 1), ischemic stroke cases had higher mean age and
40
Cerebrovasc Dis 2013;36:38-46 DOI: $10.1159 / 000351205$
Yamagishi/Folsom/Steffen/ for the ARIC Study Investigators 
Table 2. Age and sex-adjusted HRs and 95\% CIs of incident ischemic stroke for the highest and lowest quartiles of each fatty acid; ARIC Study, Minneapolis field center, 1987-2008

\begin{tabular}{|c|c|c|c|c|}
\hline & \multicolumn{4}{|l|}{ Ischemic stroke } \\
\hline & \multicolumn{2}{|l|}{ CE fraction } & \multicolumn{2}{|l|}{ PL fraction } \\
\hline SFA & $1.93(1.23-3.04)$ & 0.01 & $1.64(1.05-2.57)$ & 0.03 \\
\hline Myristic (14:0) & $1.43(0.92-2.22)$ & 0.14 & $1.29(0.83-2.01)$ & 0.26 \\
\hline Stearic $(18: 0)$ & $0.92(0.60-1.42)$ & 0.71 & $1.17(0.74-1.84)$ & 0.42 \\
\hline MUFA & $1.49(0.97-2.28)$ & 0.03 & $1.42(0.92-2.18)$ & 0.15 \\
\hline Palmitoleic $(16: 1, \omega 7)$ & $1.86(1.20-2.87)$ & 0.003 & $1.52(0.99-2.34)$ & 0.005 \\
\hline Oleic $(18: 1, \omega 9)$ & $1.58(1.04-2.41)$ & 0.08 & $1.38(0.88-2.15)$ & 0.20 \\
\hline$\omega-6$ PUFA & $0.74(0.49-1.12)$ & 0.09 & $0.93(0.60-1.45)$ & 0.40 \\
\hline Linoleic $(18: 2, \omega 6)$ & $0.64(0.43-0.97)$ & 0.13 & $0.69(0.45-1.05)$ & 0.24 \\
\hline$\alpha$-Linolenic $(18: 3, \omega 3)$ & $1.14(0.76-1.72)$ & 0.61 & $1.29(0.82-2.02)$ & 0.16 \\
\hline Long-chain $\omega-3$ PUFA & $1.08(0.70-1.66)$ & 0.88 & $0.85(0.55-1.29)$ & 0.23 \\
\hline Eicosapentaenoic $(20: 5, \omega 3)$ & $1.16(0.76-1.76)$ & 0.39 & $1.18(0.78-1.78)$ & 0.37 \\
\hline Docosahexaenoic $(22: 6, \omega 3)$ & $0.70(0.45-1.08)$ & 0.07 & $0.69(0.46-1.06)$ & 0.08 \\
\hline$\Delta$-9-desaturase index $(16: 1, \omega 7 / 16: 0)$ & $1.72(1.10-2.70)$ & 0.004 & $1.62(1.04-2.53)$ & 0.007 \\
\hline$\Delta$-6-desaturase index ${ }^{1}$ & $1.37(0.89-2.10)$ & 0.15 & $1.19(0.78-1.81)$ & 0.35 \\
\hline$\Delta$-5-desaturase index $(20: 4, \omega 6 / 20: 3, \omega 6)$ & $0.85(0.54-1.32)$ & 0.37 & $0.84(0.55-1.29)$ & 0.49 \\
\hline Elongase index (18:0/16:0) & $0.85(0.54-1.36)$ & 0.47 & $0.86(0.55-1.36)$ & 0.44 \\
\hline
\end{tabular}

${ }^{1} \Delta$-6-desaturase indices were defined as $18: 3, \omega 6 / 18: 2, \omega 6$ for CE and as 20:3, $\omega 6 / 18: 2, \omega 6$ for PL.

triglycerides and lower HDL cholesterol at baseline, and for women, higher total cholesterol. Those who developed ischemic stroke were more often hypertensive and diabetic (men and women), and smokers (men only).

Since mean fatty acid composition at baseline did not differ much by sex [20], and no interaction between sex and any fatty acids in relation to ischemic stroke was observed, analyses were performed for men and women together. Table 2 presents age and sex-adjusted HRs of ischemic stroke for the highest versus lowest quartiles of each fatty acid. Greater plasma total SFA were associated with higher risk of ischemic stroke ( $\mathrm{HR}=1.93$ for the $\mathrm{CE}$ fraction and 1.64 for the PL fraction). An increased risk was also observed for MUFA, particularly for palmitoleic acid $(\mathrm{HR}=1.86$ for the CE fraction and 1.52 for the PL fraction). Higher plasma levels of $\omega-6$ PUFA were not significantly associated with reduced risks of ischemic stroke, but linoleic acid was associated with $36 \%$ lower risk of ischemic stroke in the highest versus lowest quartiles for the $\mathrm{CE}$ fraction, although $\mathrm{p}$ values for trend were not statistically significant. Lower risks of ischemic stroke were not observed for greater total $\omega$-3 PUFA, $\alpha$-linolenic or eicosapentaenoic acids. However, being in the highest versus lowest quartile of docosahexaenoic acid was associated with approximately $30 \%$ less risk of ischemic stroke, with marginal statistical significance ( $p$ for trend $=0.07$ for CE and 0.08 for PL fractions). The $\Delta-9$ desaturase index was associated positively with ischemic stroke incidence. There was also a positive, but marginal, association for $\Delta$-6-desaturase index. No significant associations were found for $\Delta-5$-desaturase and elongase indices.

These associations did not change materially with further adjustment for cardiovascular risk factors including smoking, cigarette-years and alcohol consumption.

The positive linear associations between ischemic stroke and total SFA (fig. 1a) and palmitoleic acid (online suppl. fig. 1e; for all online suppl. material, see www. karger.com/doi/10.1159/000351205) remained statistically significant for both the CE and PL fractions. The 
Fig. 1. a Multivariable adjusted HRs and 95\% CIs of ischemic stroke according to quartiles of plasma SFA. Left: CE fraction ( $p$ for linear trend across the quartiles $=0.008$ ), right: PL fraction ( $\mathrm{p}$ for linear trend across the quartiles $=0.02)$. b Multivariable adjusted HRs and 95\% CIs of ischemic stroke according to quartiles of plasma MUFA. Left: CE fraction ( $\mathrm{p}$ for linear trend across the quartiles $=0.08)$, right: $\mathrm{PL}$ fraction $(\mathrm{p}$ for linear trend across the quartiles $=0.47$ ).

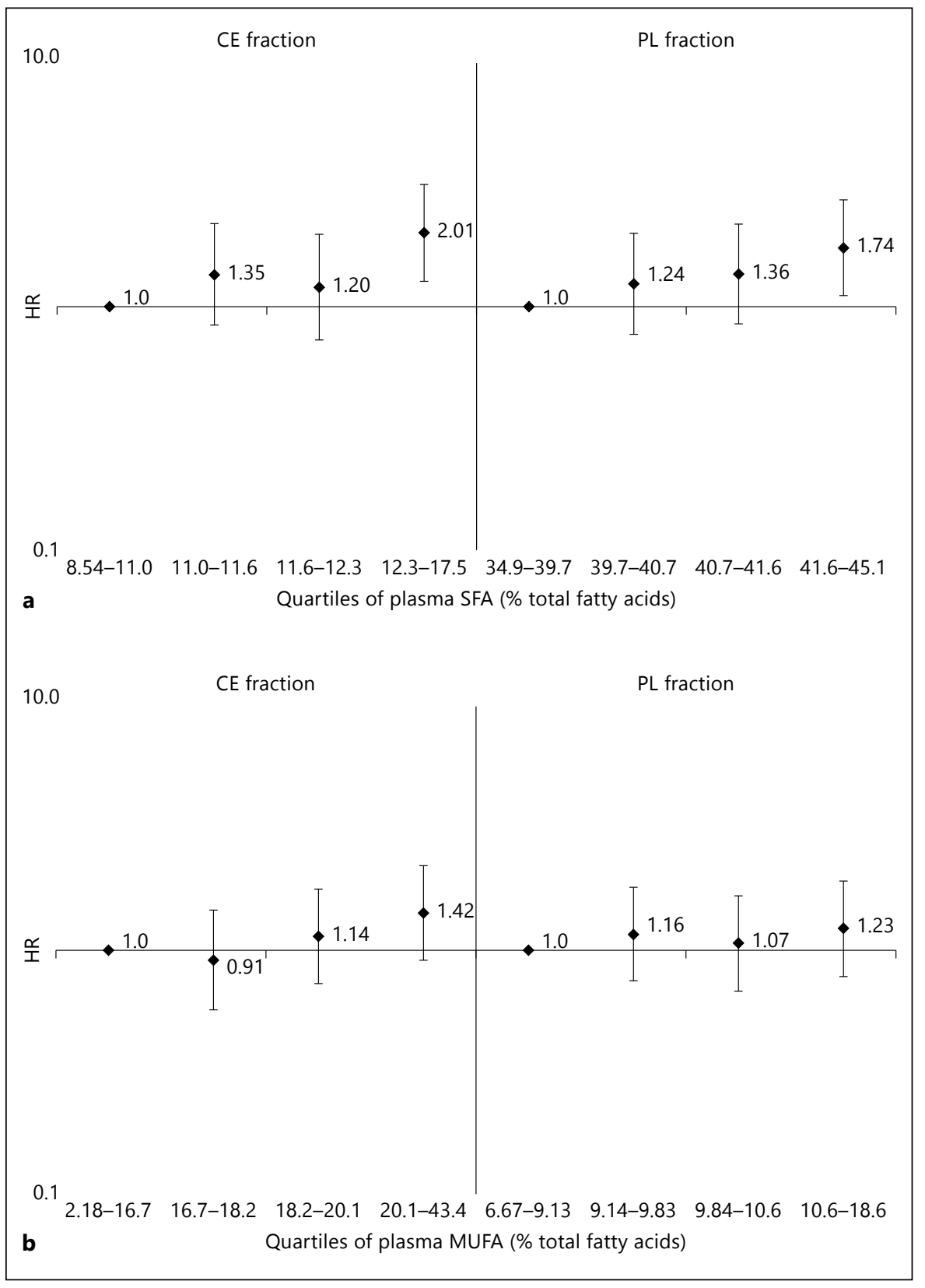

associations of MUFA (fig. 1b), $\omega-6$ PUFA (fig. 1c) and docosahexaenoic acid (online suppl. fig. $1 \mathrm{~g}$ ) with ischemic stroke were attenuated. A nonlinear inverse association between linoleic acid and ischemic stroke was still observed (online suppl. fig. 1f). No association was found for total long-chain $\omega-3$ PUFA (fig. 1d). For enzyme indices, the ischemic stroke associations were generally unchanged, and that for the $\Delta$-9-desaturation index for CE fraction was still statistically significant: HRs were 1.0 for the lowest quartile, 1.09 (0.67-1.76) for the second quar- tile, $1.75(1.11-2.74)$ for the third quartile and 1.93 (1.203.10) for the highest quartile, $\mathrm{p}$ for linear trend $=0.002$ (not shown in figures).

\section{Discussion}

By and large, the results were consistent with previous prospective studies of ischemic stroke and fatty acid biomarkers [5-8], which reported positive associations of
Yamagishi/Folsom/Steffen/ for the ARIC Study Investigators 
Fig. 1. c Multivariable adjusted HRs and 95\% CIs of ischemic stroke according to quartiles of plasma $\omega-6$ PUFA. Left: CE fraction ( $\mathrm{p}$ for linear trend across the quartiles $=0.13$ ), right: PL fraction ( $\mathrm{p}$ for linear trend across the quartiles $=0.55)$. d Multivariable adjusted HRs and 95\% CIs of ischemic stroke according to quartiles of plasma long-chain $\omega-3$ PUFA. Left: CE fraction ( $\mathrm{p}$ for linear trend across the quartiles = 0.52 ), right: PL fraction ( $\mathrm{p}$ for linear trend across the quartiles $=0.51$ ).

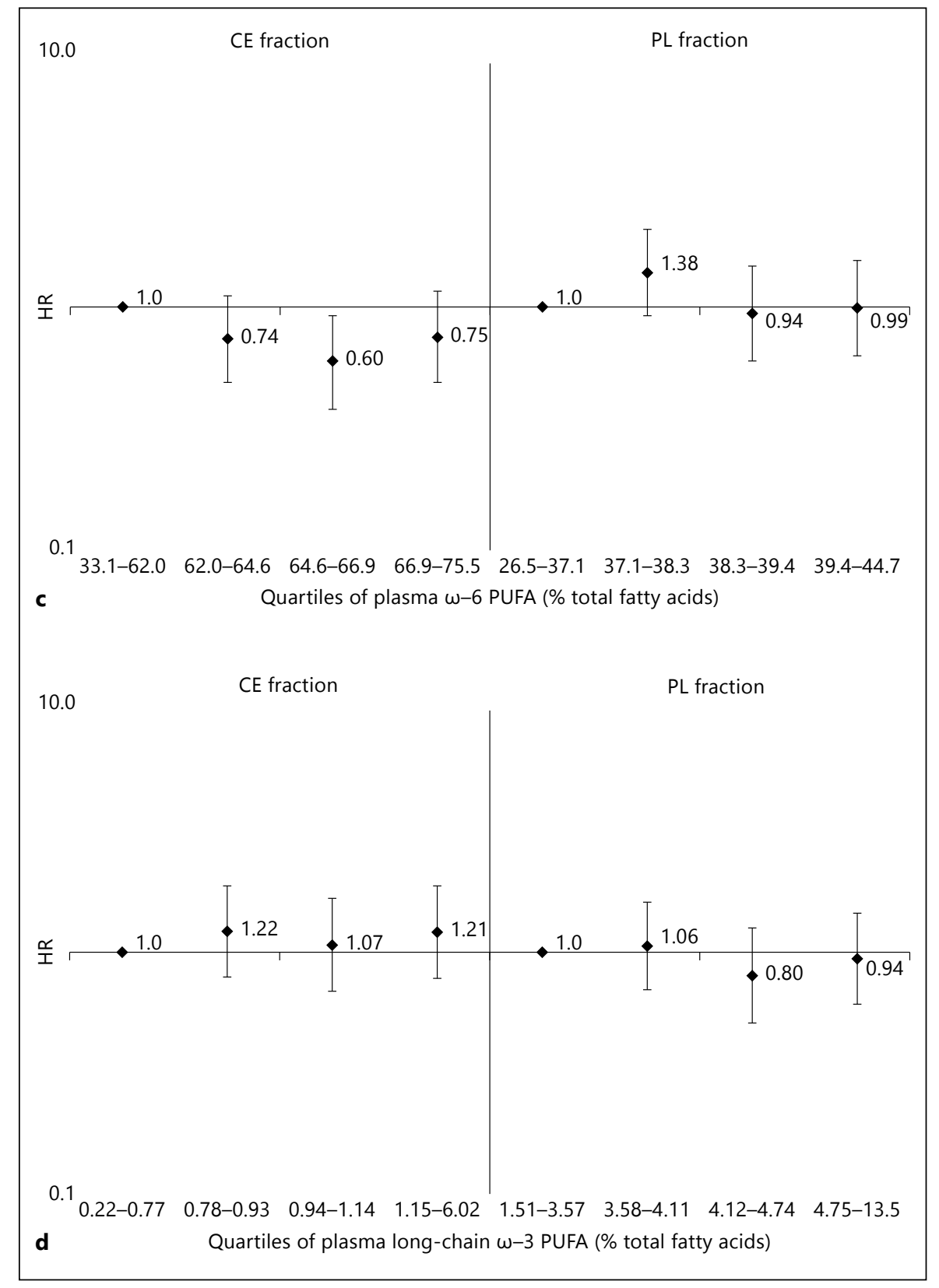

SFA and palmitoleic acid and an inverse association of linoleic acid with ischemic or total stroke, although some of these associations were attenuated after adjustment for other risk factors. Some inconsistencies among studies could be partly explained: (1) by whether studies were adjusted or not for factors potentially on the causal pathway (e.g. hypertension and lipids) or (2) by different proportions of stroke subtypes (large-artery occulusive, lacunar and hemorrhagic) across the studies. For example, $78 \%$ of ischemic strokes were lacunar in a study of
Japanese [6], while definite lacunar infarction was only $20 \%$ among these ARIC white Minnesotans. Some other fatty acid studies of whites did not classify stroke into ischemic and hemorrhagic types $[5,8]$, but would be expected to involve mostly ischemic. The tissues studied, fatty acid assay methods, distribution of fatty acids among populations, and endpoint determinations were also not equivalent across the studies.

We found a relatively strong significant positive association of both CE and PL SFA with ischemic stroke. Al- 
though there have been no dietary studies showing positive associations between SFA intake and stroke [21-26], and some reported even inverse associations with ischemic stroke [21, 24, 26, 27], a different finding for biomarkers is not a surprise. Plasma SFA is affected by endogenous synthesis, but self-report dietary intake is known for its measurement error [28]. A study in Scotland [8] reported an inverse association between SFA in adipose tissue and stroke, but they included hemorrhagic stroke in the stroke endpoint, which has been reported to be inversely associated with SFA in dietary studies $[27,29$, 30]. A Swedish study reported positive associations of myristic and palmitic acids with ischemic stroke/transient ischemic attack [7]. A Japanese nested case-control study found a positive association of serum SFA with incident ischemic stroke (predominantly lacunar stroke), but they noted the association was confounded by linoleic acid, and only linoleic acid showed a significant inverse association after multiple adjustment [6]. We speculate that SFA may have an adverse impact on nonlacunar thrombotic stroke more strongly than lacunar stroke, because an adverse impact of total or LDL cholesterol has been established only for nonlacunar thrombotic infarction and not lacunar infarction $[31,32]$. This could partly explain the relatively strong association of SFA with ischemic stroke in the present study involving a low proportion of lacunar strokes. Some inconsistency with Japanese dietary studies, which showed inverse associations between dietary SFA and ischemic stroke [26, 27], could also be explained by a large difference in distribution of SFA intake between the US and Japanese populations [33, 34].

We observed a strong positive association of MUFA, especially palmitoleic acid, as well as that of $\Delta$-9-desaturase index, with ischemic stroke. It is unlikely that plasma MUFA is a well-reflected dietary MUFA intake since it did not correlate with diet $(\mathrm{r}=0.01$ for $\mathrm{CE}$ and 0.05 for PL) [15]. Rather, plasma MUFA may reflect both SFA intake and $\Delta$-9-desaturase activity, since much of plasma MUFA is derived from SFA desaturated by $\Delta$-9-desaturase [35].

Some previous studies reported inverse associations of linoleic acid with incident ischemic stroke $[6,7]$. This was also replicated here, although the association was nonlinear. On the other hand, no association of ischemic stroke with $\omega-3$ PUFA, either $a$-linolenic or eicosapentaenoic acid, was observed in this cohort of white subjects, although a weak inverse association was detected for docosahexaenoic acid. One of the reasons for no associations for $\omega-3$ PUFA could be the very low proportion of these fatty acids in this Minnesotan population. Mean proportions of $\alpha$-linolenic, eicosapentaenoic and docosahexaenoic acids at baseline were $0.4,0.5$ and $0.4 \%$, respectively, for the $\mathrm{CE}$ fraction and $0.1,0.6$ and $2.8 \%$, respectively, for the PL fraction.

Previous studies showed that high $\Delta$-9-desaturase index, an estimate of $\Delta$-9-desaturase (stearoyl-coenzyme A desaturase) activity, has been associated with obesity and mortality from cardiovascular disease. [36, 37] Increased $\Delta$-9-desaturase activity has been demonstrated with higher SFA intake with dietary intervention [38]. In the present study, risk of ischemic stroke was greater with a higher $\Delta$-9-desaturase index defined by a ratio of 16:1/16:0. Potential mediating mechanisms between fatty acid intake and risk of stroke include high total cholesterol, obesity, inflammation and endothelial dysfunction $[39,40]$.

A strength of the present study was the use of a biomarker, which is a more objective indicator of dietary fatty acid intake than a self-report dietary questionnaire. On the other hand, plasma fatty acids do not fully reflect dietary intake, since they are also influenced by preferential incorporation, endogenous synthesis, genetics or lifestyle [11]. Plasma $\omega-3$ PUFA, especially docosahexaenoic acid, correlated well with dietary intake ( $\mathrm{r}=0.42$ for $\mathrm{CE}$ and 0.42 for PL docosahexaenoic acid), whereas SFA correlated moderately with diet, but MUFA did not [15].

We did not adjust for blood pressure, diabetes or lipids because we considered them possible mediators of any association between fatty acids and ischemic stroke. However, these are time-varying covariates and as such they may be confounders as well as mediators on the causal pathway. When we further adjusted for systolic blood pressure, antihypertensive medication use, diabetes and total and HDL cholesterol, as well as age, smoking, cigarette-years and alcohol consumption, the associations with stroke were generally attenuated for SFA and palmitoleic and linoleic acids. Other potential residual confounders, such as socioeconomic status, might have also affected the results. Another limitation is that the short- and long-term repeatability of some plasma fatty acids was modest, especially those composing low proportions of total fatty acids, including $\alpha$-linolenic acid $(\mathrm{r}=0.32-0.50$ for short-term and $0.35-0.41$ for longterm) and docosahexaenoic acid $(\mathrm{r}=0.53-0.58$ for shortterm and $0.46-0.48$ for long-term), although the repeatability of most major fatty acids (e.g. palmitic, stearic, palmitoleic, linoleic and arachidonic acids) were fair ( $\mathrm{r}>$ 0.65) [16].

In conclusion, we found significant positive associations of SFAs and MUFAs, especially palmitoleic acid in
Yamagishi/Folsom/Steffen/ for the ARIC Study Investigators 
plasma CE and PL, with incident ischemic stroke and an inverse nonlinear association between plasma linoleic acid and ischemic stroke among whites in Minnesota. As for clinical and public health implications, high dietary SFA intake and low linoleic acid intake could be risk factors for ischemic stroke, given at least modest correlations with diet and fair repeatability for these plasma fatty acids. Further investigation, including meta-analyses and clinical trials, would be needed to confirm this finding.

\section{Acknowledgments}

The ARIC Study is carried out as a collaborative study supported by National Heart, Lung, and Blood Institute contracts (HHSN268201100005C, HHSN268201100006C, HHSN26820110
0007C, HHSN268201100008C, HHSN268201100009C, HHSN268 201100010C, HHSN268201100011C and HHSN268201100012C). This study was also supported by the National Institute of Health Research Project Grant Program (R01 HL40848). Kazumasa Yamagishi was supported by the Institutional Program for Young Researcher Overseas Visits, Japan Society for the Promotion of Sciences, Japan.

The authors thank the staff and participants of the ARIC study for their important contributions. The authors also thank Linda Lewis for analyzing the plasma fatty acids.

\section{Disclosure Statement}

None of the authors had a personal or financial conflict of interest.

\section{References}

1 Erkkilä A, de Mello VD, Riserus U, Laaksonen DE: Dietary fatty acids and cardiovascular disease: an epidemiological approach. Prog Lipid Res 2008;47:172-187.

2 Wang C, Harris WS, Chung M, Lichtenstein AH, Balk EM, Kupelnick B, Jordan HS, Lau $\mathrm{J}: \mathrm{n}-3$ fatty acids from fish or fish oil supplements, but not $\alpha$-linolenic acid, benefit cardiovascular disease outcomes in primaryand secondary-prevention studies: a systematic review. Am J Clin Nutr 2006;84 5-17.

3 Siri-Tarino P, Sun Q, Hu F, Krauss R: Metaanalysis of prospective cohort studies evaluating the association of saturated fat with cardiovascular disease. Am J Clin Nutr 2010;91: 535-546.

-4 Feigin VL, Anderson CS, Ní Mhurchu C: Systemic inflammation, endothelial dysfunction, dietary fatty acids and micronutrients as risk factors for stroke: a selective review. Cerebrovasc Dis 2002;13:219-224.

-5 Simon JA, Fong J, Bernert JT Jr, Browner WS: Serum fatty acids and the risk of stroke. Stroke 1995;26:778-782.

-6 Iso H, Sato S, Umemura U, Kudo M, Koike K, Kitamura A, Imano H, Okamura T, Naito Y, Shimamoto T: Linoleic acid, other fatty acids, and the risk of stroke. Stroke 2002;33:20862093.

7 Wiberg B, Sundström J, Árnlöv J, Terént A, Vessby B, Zethelius B, Lind L: Metabolic risk factors for stroke and transient ischemic attacks in middle-aged men: a communitybased study with long-term follow-up. Stroke 2006;37:2898-2903.

8 Woodward M, Tunstall-Pedoe H, Batty GD, Tavendale R, Hu FB, Czernichow S: The prognostic value of adipose tissue fatty acids for incident cardiovascular disease: results from 3,944 subjects in the Scottish Heart Health
Extended Cohort Study. Eur Heart J 2011;32 1416-1423.

-9 Riboli E, Ronnholm H, Saracci R: Biological markers of diet. Cancer Surv 1987;6:685-718.

10 Vessby B, Gustafsson IB, Tengblad S, Boberg $M$, Andersson A: Desaturation and elongation of fatty acids and insulin action. Ann NY Acad Sci 2002;967:183-195

-11 Hodson L, Skeaff CM, Fielding BA: Fatty acid composition of adipose tissue and blood in humans and its use as a biomarker of dietary intake. Prog Lipid Res 2008;47:348-380.

12 Warensjö E, Risérus U, Vessby B: Fatty acid composition of serum lipids predicts the development of the metabolic syndrome in men. Diabetologia 2005;48:1999-2005.

13 The ARIC Investigators: The Atherosclerosis Risk in Communities (ARIC) Study: design and objectives. Am J Epidemiol 1989;129: 687-702.

14 Matthan NR, Ip B, Resteghini N, Ausman LM Lichtenstein AH: Long-term fatty acid stability in human serum cholesteryl ester, triglyceride, and phospholipid fractions. J Lipid Res 2010;51:2826-2832.

15 Ma J, Folsom AR, Shahar E, Eckfeldt JH: Plasma fatty acid composition as an indicator of habitual dietary fat intake in middle-aged adults. The Atherosclerosis Risk in Communities (ARIC) Study Investigators. Am J Clin Nutr 1995;62:564-571.

16 Ma J, Folsom AR, Eckfeldt JH, Lewis L, Chambless LE: Short- and long-term repeatability of fatty acid composition of human plasma phospholipids and cholesterol esters. The Atherosclerosis Risk in Communities (ARIC) Study Investigators. Am J Clin Nutr 1995;62:572-578.

17 Walker AE, Robins M, Weinfeld FD: The National Survey of Stroke. Clinical findings. Stroke 1981;12:I13-I44.
18 Rosamond WD, Folsom AR, Chambless LE, Wang CH, McGovern PG, Howard G, Copper LS, Shahar E: Stroke incidence and survival among middle-aged adults: 9-year follow-up of the Atherosclerosis Risk in Communities (ARIC) cohort. Stroke 1999;30: 736-743.

19 Okada T, Furuhashi N, Kuromori Y, Miyashita M, Iwata F, Harada K: Plasma palmitoleic acid content and obesity in children. Am J Clin Nutr 2005;82:747-750.

20 Yamagishi K, Nettleton JA, Folsom AR: Plasma fatty acid composition and incident heart failure in middle-aged adults: the Atherosclerosis Risk in Communities (ARIC) Study. Am Heart J 2008;156:965-974.

21 Gillman MW, Cupples LA, Millen BE, Ellison RC, Wolf PA: Inverse association of dietary fat with development of ischemic stroke in men. JAMA 1997;278:2145-2150.

22 Seino F, Date C, Nakayama T, Yoshiike N, Yokoyama T, Yamaguchi M, Tanaka H: Dietary lipids and incidence of cerebral infarction in a japanese rural community. J Nutr Sci Vitaminol 1997;43:83-99.

-23 He K, Merchant A, Rimm EB, Rosner BA Stampfer MJ, Willett WC, Ascherio A: Dietary fat intake and risk of stroke in male us healthcare professionals: 14-year prospective cohort study. BMJ 2003;327:777-782.

-24 Sauvaget C, Nagano J, Hayashi M, Yamada M Animal protein, animal fat, and cholesterol intakes and risk of cerebral infarction mortality in the adult health study. Stroke 2004;35: 1531-1537.

25 Leosdottir M, Nilsson PM, Nilsson JÅ, Berglund G: Cardiovascular event risk in relation to dietary fat intake in middle-aged individuals: data from the Malmö Diet and Cancer Study. Eur J Cardiovasc Prev Rehabil 2007;14: 701-706. 
26 Yamagishi K, Iso H, Yatsuya H, Tanabe N, Date C, Kikuchi S, Yamamoto A, Inaba Y, Tamakoshi A, JACC Study Group: Dietary intake of saturated fatty acids and mortality from cardiovascular disease among Japanese: the JACC Study. Am J Clin Nutr 2010;92:759765.

27 Yamagishi K, Iso H, Kokubo Y, Saito I, Yatsuya $\mathrm{H}$, Ishihara J, Inoue A, Tsugane S, JPHC Study Group: Dietary intake of saturated fatty acids and incident stroke and coronary heart disease in Japanese communities: the JPHC Study. Eur Heart J 2013;34:12251232.

28 Poppitt S, Swann D, Black A, Prentice A: Assessment of selective under-reporting of food intake by both obese and non-obese women in a metabolic facility. Int J Obes Relat Metab Disord 1998;22:303-311.

-29 Iso H, Stampfer MJ, Manson JE, Rexrode K, Hu FB, Hennekens CH, Colditz GA, Speizer FE, Willett WC: Prospective study of fat and protein intake and risk of intraparenchymal hemorrhage in women. Circulation 2001; 103 : 856-863.
30 Iso H, Sato S, Kitamura A, Naito Y, Shimamoto T, Komachi Y: Fat and protein intakes and risk of intraparenchymal hemorrhage among middle-aged Japanese. Am J Epidemiol 2003;157:32-39.

31 Ohira T, Shahar E, Chambless LE, Rosamond WD, Mosley TH Jr, Folsom AR: Risk factors for ischemic stroke subtypes: the Atherosclerosis Risk in Communities Study. Stroke 2006;37:2493-2498.

32 Imamura T, Doi Y, Arima H, Yonemoto K, Hata J, Kubo M, Tanizaki Y, Ibayashi S, Iida M, Kiyohara Y: LDL cholesterol and the development of stroke subtypes and coronary heart disease in a general Japanese population: the Hisayama Study. Stroke 2009;40:382-388.

33 Muramatsu K, Tsuchihashi N, Tanaka E, Yamaguchi M, Suzuki A, Ishii K, Watanabe T: Estimated intake of cholesterol and fatty acids in Japanese. Bull Chiba Coll Health Sci 2004; 23:1-25 (in Japanese).

34 Kennedy ET, Bowman SA, Powell R: Dietaryfat intake in the US population. J Am Coll Nutr 1999;18:207-212.

35 Vessby B: Dietary fat, fatty acid composition in plasma and the metabolic syndrome. Curr Opin Lipidol 2003;14:15-19.
36 Warensjö E, Ohrvall M, Vessby B: Fatty acid composition and estimated desaturase activities are associated with obesity and lifestyle variables in men and women. Nutr Metab Cardiovasc Dis 2006;16:128-136.

37 Warensjö E, Sundström J, Vessby B, Cederholm T, Risérus U: Markers of dietary fat quality and fatty acid desaturation as predictors of total and cardiovascular mortality: a population-based prospective study. Am J Clin Nutr 2008;88:203-209.

38 Warensjö E, Risérus U, Gustafsson I, Mohsen R, Cederholm T, Vessby B: Effects of saturated and unsaturated fatty acids on estimated desaturase activities during a controlled dietary intervention. Nutr Metab Cardiovasc Dis 2008; 18:683-690.

39 Sacks F, Katan M: Randomized clinical trials on the effects of dietary fat and carbohydrate on plasma lipoproteins and cardiovascular disease. Am J Med 2002;113:13S-24S.

40 De Caterina R, Zampolli A, Del Turco S, Madonna R, Massaro M: Nutritional mechanisms that influence cardiovascular disease. Am J Clin Nutr 2006;83:421S-426S. 